\title{
Index Coding via Random Coding
}

\author{
Fatemeh Arbabjolfaei *, Bernd Bandemer ${ }^{\dagger}$, Young-Han Kim* \\ * Department of Electrical and Computer Engineering \\ University of California, San Diego \\ ${ }^{\dagger}$ Bosch Research and Technology Center North America
}

\begin{abstract}
The index coding problem is a simple distributed source coding problem in which a sender broadcasts multiple messages to their respective receivers with side information about other messages. This problem arises in many applications such as content broadcasting, distributed caching, and wireless interference management. At the same time, it is a canonical instance of the multipleunicast network coding problem that captures the essence of broadcasting multiple interfering streams. Reflecting the importance as well as the difficulty of the index coding problem, several coding schemes have been proposed that are built on tools from graph theory, linear network coding, combinatorial optimization, and interference alignment. This paper studies the composite coding scheme based on random coding in information theory. Despite its conceptual simplicity that allows for rather straightforward analysis, the scheme uniformly outperforms the existing coding schemes by Birk and Kol (1998), Blasiak, Kleinberg, and Lubetzky (2013), and Shanmugam, Dimakis, and Langberg (2013), and is optimal for all index coding problems with up to five messages.
\end{abstract}

\section{INTRODUCTION}

Consider a communication scenario in which a sender has multiple messages to communicate to a set of receivers. Each receiver is interested in one of the messages and has prior side information consisting of a subset of the other messages. What is the amount of information that should be broadcasted from the server to the receivers so that every receiver can recover its desired message? This problem, broadly referred to as index coding, is one of the canonical problems that capture the effect of broadcast and interference in multiuser communication systems. Originally introduced by Birk and $\mathrm{Kol}$ [1], [2] in the context of satellite communication, the index coding problem has received significant attention from various disciplines such as theoretical computer science [3]-[6], network coding [7], [8], information theory [9], [10], and wireless communication [11], [12].

There are many variants of the problem considered in the literature. In this paper, we consider the following variant with large data blocks that was initially formalized by Alon, Lubetzky, Stav, Weinstein, and Hassidim [5] and studied in most subsequent papers. Suppose that a sender wishes to broadcast a tuple of $n$ messages, $x^{n}=\left(x_{1}, \ldots, x_{n}\right)$, where $x_{i} \in\{0,1\}^{t}$ for all $i$, to $n$ receivers. Receiver $j \in[n]:=\{1,2, \ldots, n\}$ has prior knowledge of $x\left(\mathcal{A}_{j}\right):=\left(x_{i}: i \in \mathcal{A}_{j}\right)$, where $\mathcal{A}_{j} \subseteq[n] \backslash\{j\}$, and wishes to recover $x_{j}$. Assume that the sender is aware of $\mathcal{A}_{1}, \ldots, \mathcal{A}_{n}$.

Since an index coding problem is fully characterized by the side information sets $\mathcal{A}_{1}, \ldots, \mathcal{A}_{n}$, as a shorthand notation we write the problem as $\left(1 \mid \mathcal{A}_{1}\right), \ldots,\left(n \mid \mathcal{A}_{n}\right)$. For example, the 3-message index coding problem with $\mathcal{A}_{1}=\{2\}, \mathcal{A}_{2}=\{1,3\}$, and $\mathcal{A}_{3}=\{1\}$ can be written as $(1 \mid 2),(2 \mid 1,3),(3 \mid 1)$. An index coding problem can be also represented by a directed graph $\mathcal{G}=(\mathcal{V}, \mathcal{E})$, referred to as the side information graph, ${ }^{1}$ where $\mathcal{V}=[n]$ and $(i, j) \in \mathcal{E}$ iff $i \in \mathcal{A}_{j}$; see Figure 1 for an illustration.

Given an index coding problem, a $t$-bit index code $\mathcal{C}=\left(\phi,\left\{\psi_{j}\right\}\right)$ is defined by

- an encoder $\phi:\{0,1\}^{n t} \rightarrow\{0,1\}^{r}$ that maps $n$ tuple of $t$-bit messages to an $r$-bit index, and

- $n$ decoders $\psi_{j}:\{0,1\}^{r} \times\{0,1\}^{\left|\mathcal{A}_{j}\right| t} \rightarrow\{0,1\}^{t}$ that maps the received string $y=\phi\left(x^{n}\right)$ and the side information $x\left(\mathcal{A}_{j}\right)$ to a $t$-bit message estimate.

The performance of a code $\mathcal{C}$ is measured by its rate $\beta(\mathcal{C})=r / t$ and the average probability of decoding error

$$
\alpha(\mathcal{C})=\frac{1}{2^{n t}} \sum_{x^{n}} \mathbf{1}_{\left\{\psi_{j}\left(\phi\left(x^{n}\right), x\left(\mathcal{A}_{j}\right)\right) \neq x_{j} \text { for some } j\right\}},
$$

that is, the probability that at least one receiver does not recover its desired message correctly. Define the optimal broadcast rate of the index coding problem as

$$
\beta^{*}=\inf _{t} \inf _{\mathcal{C}: \alpha(\mathcal{C})=0} \beta(\mathcal{C}),
$$

where the second infimum is over all $t$-bit index codes that achieve zero decoding error, i.e., $\phi$ is one-to-one for each $x_{j}$ given $x\left(\mathcal{A}_{j}\right)$. Thus, $\beta^{*}$ characterizes the fundamental limit on the rate of index codes such that every message can be recovered exactly. Further define

$$
\beta_{\epsilon}^{*}=\inf _{t} \inf _{\mathcal{C}: \alpha(\mathcal{C}) \leq \epsilon} \beta(\mathcal{C}) .
$$

Under this notation, $\beta^{*}=\beta_{0}^{*}$. Furthermore, the following relationship holds between $\beta^{*}$ and $\beta_{\epsilon}^{*}$.

\footnotetext{
${ }^{1}$ Some papers use the opposite convention in which the directions of the edges are reversed.
} 
Lemma 1 (Langberg and Effros [13]). $\beta^{*}=\lim _{\epsilon \rightarrow 0} \beta_{\epsilon}^{*}$.

In light of this lemma, $\beta^{*}$ can be alternatively characterized as the infimum of the rates $\beta$ such that there exists a sequence of $t$-bit index codes $\left\{\mathcal{C}_{t}\right\}_{t=1}^{\infty}$ such that $\beta\left(\mathcal{C}_{t}\right)=\beta$ and $\lim _{t \rightarrow \infty} \alpha\left(\mathcal{C}_{t}\right)=0$.

An important application of the index coding problem is content broadcasting, in which a server communicates data packets to multiple clients. In the first phase of communication, the server broadcasts the packets and each client stores only a subset of the transmitted packets due to communication error or limited storage. In the second phase of communication, index coding can be used to reduce the number of required transmissions. This situation arises in satellite communication [1] or peer-to-peer video distribution [5]; see also [14] for a more holistic analysis on distributed caching.

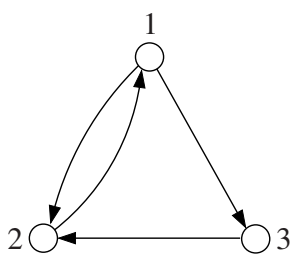

(a)

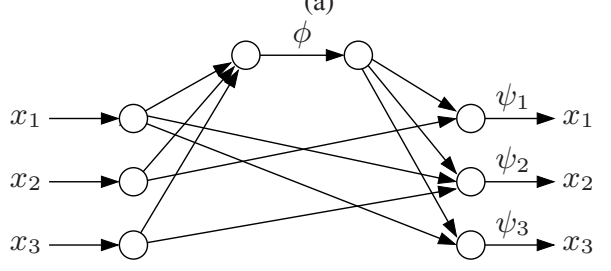

(b)

Fig. 1. (a) The directed graph representation for the index coding problem $(1 \mid 2),(2 \mid 1,3),(3 \mid 1)$. (b) The equivalent network coding problem.

Another application is wireless network interference management, in which multiple interfering transmitters communicate to their respective receivers. Suppose that due to the topology of the wireless network, each receiver has only a subset of signals interfering with the desired signal. Then, we can construct a corresponding index coding problem by taking the noninterfering signals as the side information at the receiver. In [11], [12], it is shown that the symmetric degrees of freedom (the symmetric capacity at high signal-to-noise ratio) is upper bounded by the symmetric capacity (the reciprocal of the optimal broadcast rate $\beta^{*}$ ) of the corresponding index coding problem and the two problems are equivalent under linear codes.

Perhaps the most important motivation for index coding arises in network coding. As illustrated in Figure 1, index coding is a representative instance of the multipleunicast network coding problem, in which there is a single bottleneck link that can utilize the index code for the given side information pattern. Moreover, Effros, El Rouayheb, and Langberg [8] showed that the zero-error capacity region of any multiple network coding problem can be found by characterizing $\beta^{*}$ of a corresponding index coding problem. Thus, any progress in the index coding problem can lead to advancement in network coding.

In this paper, we first present an upper bound on the optimal broadcast rate of a general index coding problem using the composite coding scheme outlined in [15]. Reminiscent of the coding scheme in the original network coding paper by Ahlswede, Cai, Li, and Yeung [16], the composite coding scheme is built on Shannon's random coding [17] (or more precisely, Cover's random binning [18]) approach that establishes the existence of a good code through an elementary probabilistic method. Rather surprisingly, this coding scheme is optimal for small $n$; for example, it achieves $\beta^{*}$ for all 9608 index coding problems of $n=5$. (See [19] for the complete list of the problems and optimal broadcast rates.)

Second, we compare the composite coding scheme with existing coding schemes. In particular, we show that the composite coding scheme uniformly outperforms the (partial) clique covering scheme by Birk and Kol [1], the fractional clique covering (graph coloring) scheme by Blasiak, Kleinberg, and Lubetzky [6], and the fractional local graph coloring scheme by Shanmugam, Dimakis, and Langberg [10].

The rest of the paper is organized as follows. In the next section, we survey the existing coding schemes in the literature. In Section III, we describe the composite coding scheme and characterize the broadcast rate $\beta_{\mathrm{CC}}$ it achieves as the solution to a linear program. In Section IV, we compare $\beta_{\mathrm{CC}}$ with the broadcast rates $\beta_{\mathrm{FL}}$ achieved by fractional local coloring and $\beta_{\mathrm{PC}}$ achieved by partial clique covering. In Section V, we conclude the paper with potential extensions of composite coding and a discussion on limitations of composite coding and other existing coding schemes.

\section{EXISTING CODING SCHEMES}

Roughly speaking, there have been two general approaches to index coding in the literature. We explain these two intimately related approaches in the next two subsections. Inter alia, we will clarify the relationships among different coding schemes that are often difficult to elucidate from the literature.

\section{A. Graph coloring}

The first (and perhaps the most natural) approach to index coding is based on partitioning of the side information graph $\mathcal{G}$ by cliques (or equivalently, coloring of the undirected complement of $\mathcal{G}$ ). We illustrate this approach through the following. 
Example 1. Consider the index coding problem represented by the side information graph $\mathcal{G}$ in Figure 2(a). Note that three cliques $\{1,2\},\{3,4\}$, and $\{5\}$ partition $\mathcal{G}$. Let $t=1$ and transmit the binary sums (parities) of all the message symbols in each clique, i.e., $\phi\left(x_{1}, x_{2}, x_{3}, x_{4}, x_{5}\right)=\left(x_{1}+x_{2}, x_{3}+x_{4}, x_{5}\right)$. Since $\phi$ is injective in $x_{j}$ given $\mathcal{A}_{j}$ for every $j$, this code achieves zero error at rate $\beta=3$, which is the chromatic number of the undirected complement $\overline{\mathcal{G}}$ of $\mathcal{G}$; see Figure 2(b).

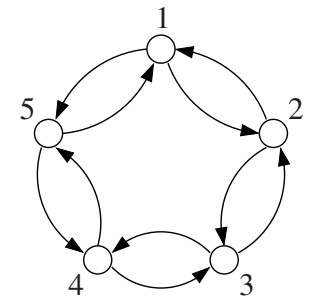

(a)

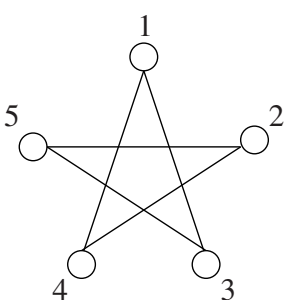

(b)
Fig. 2. (a) The directed graph $\mathcal{G}$ representing the index coding problem $(1 \mid 2,5),(2 \mid 1,3),(3 \mid 2,4),(4 \mid 3,5),(5 \mid 1,4)$. (b) Its undirected complement $\mathcal{G}$ that is obtained by drawing an undirected edge $\{i, j\}$ iff $(i, j) \notin \mathcal{E}$ or $(j, i) \notin \mathcal{E}$.

In general, for any index coding problem with side information graph $\mathcal{G}$, we can achieve the minimum number of cliques that partition $\mathcal{G}$ (or equivalently, the chromatic number of its undirected complement). It can be readily checked that this rate is the solution to the integer program

$$
\begin{aligned}
\operatorname{minimize} & \sum_{\mathcal{S} \in \mathcal{K}} \rho_{\mathcal{S}} \\
\text { subject to } & \sum_{\substack{\mathcal{S} \in \mathcal{K}: j \in \mathcal{S} \\
\rho_{\mathcal{S}} \in\{0,1\}, \quad \mathcal{S} \in \mathcal{K},}} \rho_{\mathcal{S}} \geq 1, \quad j \in[n],
\end{aligned}
$$

where $\mathcal{K}$ is the collection of all cliques in $\mathcal{G}$.

This coding scheme has been extended in several directions. First, Birk and Kol [1] showed that we can code over arbitrary (sub)graphs instead of cliques. An $n$-node directed graph is said to be a $k$-partial clique if the minimum indegree of all vertices is $n-k-1$. Suppose that the side information graph of an index coding problem is a $k$-partial clique. Then, since each receiver $j \in[n]$ is missing at most $k+1$ messages, we can transmit $k+1$ parity symbols generated from the message symbols and a systematic maximum distance separable (MDS) code [20] that can correct arbitrary $k$ erasures from them. For instance, a clique corresponds to $k=1$ and the MDS code in this case is a single parity $x_{1}+\cdots+x_{n}$. As another example, for the graph corresponding to the index coding problem $(1 \mid 2),(2 \mid 3),(3 \mid 1)$, $\mathcal{G}$ is 1 -partial clique, and two parity symbols, say, $x_{1}+x_{2}$ and $x_{1}+x_{3}$, suffice to correct any two erasures from $\left(x_{1}, x_{2}, x_{3}\right)$. Generalizing this idea to subgraphs, we can establish the following.

Theorem 1 (Birk and Kol [1]). If $\mathcal{G}_{1}, \ldots, \mathcal{G}_{m}$ are partial cliques of parameters $k_{1}, \ldots, k_{m}$ that partition $\mathcal{G}$, then the optimal broadcast rate is upper bounded by

$$
\beta_{\mathrm{PC}}\left(\mathcal{G}_{1}, \ldots, \mathcal{G}_{m}\right)=\left(k_{1}+1\right)+\cdots+\left(k_{m}+1\right)
$$

and thus by

$$
\beta_{\mathrm{PC}}=\min _{\mathcal{G}_{1}, \ldots, \mathcal{G}_{m}} \beta_{\mathrm{PC}}\left(\mathcal{G}_{1}, \ldots, \mathcal{G}_{m}\right),
$$

where the minimum is over all partitions.

Note that by limiting the subgraphs to be cliques, we recover (1).

As another extension of (1), Blasiak, Kleinberg, and Lubetzky [6] showed that we can use more than one clique per message. A function $f: 2^{[n]} \backslash\{\emptyset\} \rightarrow[0,1]$ is said to be a fractional partition if for every $j \in[n]$, $\sum_{\mathcal{S}: j \in \mathcal{S}} f(\mathcal{S})=1$. Note that a partition is a special case with $f(\mathcal{S})=0$ or 1 for every $\mathcal{S}$. Now by considering a fractional partition $f$ over all cliques (i.e., $f(\mathcal{S})>0$ only if $\mathcal{S}$ is a clique) and splitting messages according to the given fractions, we can achieve the broadcast rate $\sum_{\mathcal{S}} f(\mathcal{S})$. As an illustration, we revisit the index coding problem in Example 1. Let

$f(\mathcal{S})= \begin{cases}1 / 2, & \text { if } \mathcal{S}=\{1,2\},\{2,3\},\{3,4\},\{4,5\},\{5,1\}, \\ 0, & \text { otherwise. }\end{cases}$

It can be readily verified that $f$ is a fractional partition with $\sum f(\mathcal{S})=5 / 2$. This rate can be achieved by taking $t=2$, splitting message $x_{j}$ into $\left(x_{j 1}, x_{j 2}\right), j \in[5]$, and transmitting $\left(x_{11}+x_{21}, x_{31}+x_{41}, x_{51}+x_{12}, x_{22}+\right.$ $\left.x_{32}, x_{42}+x_{52}\right)$. In general, by optimizing over fractional partitions, we can establish the upper bound on the broadcast rate that can be rewritten as the solution to the linear program (1) with integer constraints $\rho_{\mathcal{S}} \in\{0,1\}$ relaxed by

$$
\rho_{\mathcal{S}} \in[0,1], \quad \mathcal{S} \in \mathcal{K} .
$$

This upper bound is equivalent to the fractional clique covering number of $\mathcal{G}$ or equivalently the fractional chromatic number of the undirected complement of $\mathcal{G}$.

Taking an orthogonal step to extending the original clique covering scheme, Shanmugam, Dimakis, and Langberg [10] showed that we can reduce the number of parity transmissions by applying an MDS code for parity symbols and using side information to recover them, just as Birk and Kol [1] reduced the number of message transmissions with an MDS code. For example, consider the index coding problem $(1 \mid 2,3,4),(2 \mid 1,3,4),(3 \mid 4,5,6)$, $(4 \mid 3,5,6),(5 \mid 1,2,6),(6 \mid 1,2,5)$. The side information graph $\mathcal{G}$ can be partitioned into three cliques 


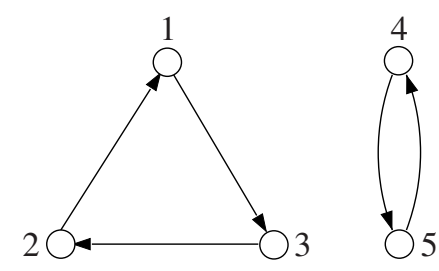

Fig. 3. The side information graph representation of the index coding problem $(1 \mid 2),(2 \mid 3),(3 \mid 1),(4 \mid 5),(5 \mid 4)$.

$\{1,2\},\{3,4\}$, and $\{5,6\}$, and hence it suffices to transmit parities $x_{1}+x_{2}, x_{3}+x_{4}$, and $x_{5}+x_{6}$. However, since only two of the three parities are missing at each receiver, we can reduce the number of parity transmissions by using a two-erasure correcting MDS code with two hyperparity symbols, say, $\left(x_{1}+x_{2}\right)+\left(x_{3}+x_{4}\right)$ and $\left(x_{1}+x_{2}\right)+\left(x_{5}+x_{6}\right)$. This coding scheme achieves the local chromatic number [21], [22] of the directed complement of $\mathcal{G}$. Further extending this scheme with fractional coloring, we can establish the following.

Theorem 2 (Shanmugam, Dimakis, and Langberg [10]). The optimal broadcast rate is upper bounded by the solution $\beta_{\mathrm{FL}}$ to the linear program

$$
\begin{array}{ll}
\operatorname{minimize} & \max _{j \in[n]} \sum_{\mathcal{S} \in \mathcal{K}: \mathcal{S} \unrhd \mathcal{A}_{j}} \rho_{\mathcal{S}} \\
\text { subject to } & \sum_{\mathcal{S} \in \mathcal{K}: j \in \mathcal{S}} \rho_{\mathcal{S}} \geq 1, \quad j \in[n], \\
& \rho_{\mathcal{S}} \in[0,1], \quad \mathcal{S} \in \mathcal{K} .
\end{array}
$$

The rate $\beta_{\mathrm{FL}}$ is referred to as the fractional local chromatic number of the directed complement of $\mathcal{G}$. Clearly, the fractional local coloring scheme outperforms the clique covering scheme (see (1)) and its fractional generalization (see (3)). Moreover, it can be shown that this relationship can be strict [10]. However, it does not outperform and is not outperformed by the partial clique covering scheme (see Theorem 1). For the index coding problem in Example 1, $\beta_{\mathrm{PC}}=3>\beta_{\mathrm{FL}}=5 / 2$. For the other direction of dominance, consider the following.

Example 2. Consider the 5-message index coding problem represented by the side information graph $\mathcal{G}$ in Figure 3. Since $\mathcal{G}$ can be partitioned into two partial cliques $\{1,2,3\}$ and $\{4,5\}$ with parameters 0 and 1 respectively, $\beta_{\mathrm{PC}}=3$. However, it can be checked that the fractional local chromatic number of the directed complement of $\mathcal{G}$ is $\beta_{\mathrm{FL}}=4$.

\section{B. Linear coding}

The coding schemes discussed in the previous subsection are all built around a special class of linear codes, namely, the MDS code for some subset of messages. Bar-Yossef, Birk, Jayram, and Kol [3] considered a general linear code. As an example, we revisit the index coding problem $(1 \mid 2),(2 \mid 3),(3 \mid 1)$ and consider any 3 by-3 matrix $M$ such that

$$
M_{i i} \neq 0 \quad \text { and } \quad M_{i j}=0 \text { if } i \notin \mathcal{A}_{j} .
$$

The rank-2 matrix

$$
M=\left(\begin{array}{lll}
1 & 0 & 1 \\
1 & 1 & 0 \\
0 & 1 & 1
\end{array}\right)
$$

satisfies such constraints. Suppose that we transmit any two independent columns of $M$ multiplied from right by the message vector $\left(x_{1}, x_{2}, x_{3}\right)$, say, $x_{1}+x_{2}$ and $x_{2}+x_{3}$. Since the matrix has rank 2 , each receiver can recover all linear combinations of the messages generated by the matrix $M$. (In coding theory, $M$ is referred to as the parity-check portion of the systematic generator matrix.) Moreover, receiver $j$ can use the linear combination from column $j$ to recover $x_{j}$ since it contains only $x_{j}$ and $x\left(\mathcal{A}_{j}\right)$. We can optimize over all matrices $M$ satisfying (5) to minimize its rank. For a general index coding problem with side information graph $\mathcal{G}$, BarYossef, Birk, Jayram, and Kol [3] defined this quantity as $\operatorname{minrk}_{2}(\mathcal{G})$, which yields an upper bound on the optimal broadcast rate. Note that this bound always holds for $t=1$.

This coding scheme has been extended in several directions. First, Lubetzky and Stav [4] showed that we can tighten the upper bound (even for $t=1$ ) by using a matrix in a larger field $\mathbb{F}$ that satisfies (5), which results in the upper bound $\left\lceil\operatorname{minrk}_{\mathbb{F}}(\mathcal{G}) \log _{2}|\mathbb{F}|\right\rceil$. For $t \gg 1$, coding over message symbols in $\mathbb{F}$ can remove the unnatural factor $\log _{2}|\mathbb{F}|$, further tightening the upper bound. Lubetzky and Stav [4] also demonstrated that for some index coding problems, the performance can be significantly improved by partitioning $\mathcal{G}$ into subgraphs and using fields of distinct characteristics over each subgraph.

Motivated by interference alignment coding schemes in wireless interference channels [23], [24], Maleki, Cadambe, and Jafar [9] proposed an extension that uses message symbols in $\mathbb{F}^{t}$ and $t$-by- $t$ matrices in place of $M_{i j}, 1 \leq i, j \leq n$, in (5). The rank of the resulting $n t-$ by-nt matrix provides an upper bound on the optimal broadcast rate.

Several special classes of index coding problems have been studied for which the scalar linear coding scheme by Bar-Yossef, Birk, Jayram, and Kol [3] or the vector linear coding scheme by Maleki, Cadambe, and Jafar [9] is optimal. In general, however, it is rather difficult to find an optimal linear code or its performance for a given index coding problem, even when $n$ is small. In this sense, the coding schemes based on graph coloring have been referred to as "the state of the art" [10]. 


\section{Composite CODING}

The basic idea of the composite coding scheme is to ensure that $\psi_{j}\left(\phi\left(x_{1}, \ldots, x_{n}\right), x\left(\mathcal{A}_{j}\right)\right)$ is one-to-one for each $j \in[n]$ with high probability by taking a composition of random maps $y_{\mathcal{S}}(x(\mathcal{S})), \mathcal{S} \subseteq[n]$, and $z\left(y_{\{1\}}, y_{\{2\}}, \ldots, y_{\mathcal{S}}, \ldots, y_{[n]}\right)$ of appropriate inputoutput rates. To illustrate the scheme, we revisit the index coding problem $(1 \mid 3),(2 \mid 1),(3 \mid 2),(4 \mid 5),(5 \mid 4)$ in Example 2 with side information graph depicted in Figure 3 and show that any broadcast rate $\beta>3$ is achievable. As the first step of composite coding, we map $\left(x_{1}, x_{2}\right)$ to an index $y_{\{1,2\}}=y_{\{1,2\}}\left(x_{1}, x_{2}\right)$ drawn uniformly at random from $\left[2^{\gamma_{\{1,2\}} t}\right]$. Similarly, map $\left(x_{2}, x_{3}\right)$ and $\left(x_{4}, x_{5}\right)$ into random indices $y_{\{2,3\}}=$ $y_{\{2,3\}}\left(x_{2}, x_{3}\right) \in\left[2^{\gamma_{\{2,3\}} t}\right]$ and $y_{\{4,5\}}=y_{\{4,5\}}\left(x_{4}, x_{5}\right) \in$ $\left[2^{\gamma_{\{4,5\}} t}\right]$, respectively. As the second step of composite coding, we map $\left(y_{\{1,2\}}, y_{\{2,3\}}, y_{\{4,5\}}\right)$ to a codeword $z=z\left(y_{\{1,2\}}, y_{\{2,3\}}, y_{\{4,5\}}\right)$ drawn uniformly at random from $\left[2^{\beta t}\right]$ and transmit it.

Decoding is also done in two steps. Each receiver $j$ first recovers $\left(y_{\{1,2\}}, y_{\{2,3\}}, y_{\{4,5\}}\right)$ from $z$, which is successful with high probability if $\gamma_{\{1,2\}}+\gamma_{\{2,3\}}+\gamma_{\{4,5\}}<$ $\beta$, and then recovers $x_{j}$ from $\left(y_{\{1,2\}}, y_{\{2,3\}}, y_{\{4,5\}}\right)$ and $x\left(\mathcal{A}_{j}\right)$. For example, receiver 1 recovers $x_{1}$ (and $x_{2}$ ) from $y_{\{1,2\}}, y_{\{2,3\}}$, and side information $x_{3}$ successfully if $\gamma_{\{1,2\}}>1$ and $\gamma_{\{2,3\}}>1$. Similarly, it can be easily verified that other receivers can recover their messages with high probability if $\gamma_{\{1,2\}}>1, \gamma_{\{2,3\}}>1$, and $\gamma_{\{4,5\}}>1$.

We can streamline this idea by letting each decoder $j$ optimize over the set $\mathcal{D}_{j}$ of messages it recovers. This establishes the following.

Theorem 3. If $\mathcal{D}_{1}, \ldots, \mathcal{D}_{n} \subseteq[n]$ satisfy

$$
j \in \mathcal{D}_{j}, \quad j \in[n],
$$

then the optimal broadcast rate is upper bounded by the solution $\beta_{\mathrm{CC}}\left(\mathcal{D}_{1}, \ldots, \mathcal{D}_{n}\right)$ to the linear program

$$
\begin{array}{ll}
\operatorname{minimize} & \max _{j \in[n]} \sum_{\mathcal{S} \subseteq[n]: \mathcal{S} \subseteq \mathcal{A}_{j}} \gamma_{\mathcal{S}} \\
\text { subject to } & \min _{\mathcal{T} \subseteq \mathcal{D}_{j} \backslash \mathcal{A}_{j}} \frac{1}{|\mathcal{T}|} \sum_{\substack{\mathcal{S} \subseteq \mathcal{D}_{j} \cup \mathcal{A}_{j} \\
\mathcal{S} \cap \mathcal{T} \neq \emptyset}} \gamma_{\mathcal{S}} \geq 1, \quad j \in[n], \\
& \gamma_{\mathcal{S}} \geq 0, \quad \mathcal{S} \subseteq[n],
\end{array}
$$

and thus by

$$
\beta_{\mathrm{CC}}=\min _{\mathcal{D}_{1}, \ldots, \mathcal{D}_{n}} \beta_{\mathrm{CC}}\left(\mathcal{D}_{1}, \ldots, \mathcal{D}_{n}\right),
$$

where the minimum is over all subset tuples $\left(\mathcal{D}_{1}, \ldots, \mathcal{D}_{n}\right)$ satisfying $(6)$.

Proof: Following the standard steps in random coding proofs in information theory [25], [26], we prove the theorem by describing a $t$-bit randomly generated index code ensemble of rate $\beta$ and showing that the average probability of error of the random code ensemble tends to zero as $t \rightarrow \infty$, provided that $\beta>\beta_{\mathrm{CC}}\left(\mathcal{D}_{1}, \ldots, \mathcal{D}_{n}\right)$ for some $\left(\mathcal{D}_{1}, \ldots, \mathcal{D}_{n}\right)$ satisfying (6).

Codebook generation. Fix $\beta>0$ and $\gamma_{\mathcal{S}} \geq 0$ for each $\mathcal{S} \subseteq[n]$. For simplicity of exposition, we assume that $\beta t$ and $\gamma_{\mathcal{S}} t$ are integers. For each $\mathcal{S}$ and each $x(\mathcal{S}) \in\left[2^{|\mathcal{S}| t}\right]$, independently generate $y_{\mathcal{S}}(x(\mathcal{S}))$ uniformly at random from $\left[2^{\gamma_{\mathcal{S}} t}\right]$. For each $\left(y_{\{1\}}, y_{\{2\}}, \ldots, y_{\mathcal{S}}, \ldots, y_{[n]}\right) \in$ $\left[2^{\gamma_{\{1\}} t}\right] \times\left[2^{\gamma_{\{2\}} t}\right] \times \cdots \times\left[2^{\gamma_{\mathcal{S}} t}\right] \times \cdots \times\left[2^{\gamma_{[n]} t}\right]$, independently generate $z\left(y_{\{1\}}, y_{\{2\}}, \ldots, y_{\mathcal{S}}, \ldots, y_{[n]}\right)$ uniformly at random from $\left[2^{\beta t}\right]$.

Encoding. To send message tuple $\left(x_{1}, \ldots, x_{n}\right)$, the encoder first computes $y_{\mathcal{S}}=y_{\mathcal{S}}(x(\mathcal{S}))$ for each $\mathcal{S} \subseteq$ $[n]$ and then transmits the corresponding codeword $z\left(y_{\{1\}}, \ldots, y_{[n]}\right)$.

Decoding at receiver $j$. As with the encoding, decoding also has two steps. First, decoder $j$ declares that $\left(\hat{y}_{\{1\}}, \ldots, \hat{y}_{[n]}\right)$ is sent if it is the unique tuple such that $z\left(\hat{y}_{\{1\}}, \ldots, \hat{y}_{[n]}\right)=z$, where $\hat{y}_{\mathcal{S}}=y_{\mathcal{S}}(x(\mathcal{S}))$ for every $\mathcal{S} \subseteq \mathcal{A}_{j}$. If there is more than one such tuple, then it declares an error.

Next, decoder $j$ declares that $\hat{x}\left(\mathcal{D}_{j} \cup \mathcal{A}_{j}\right)$ is sent if it is a unique tuple that satisfies $\hat{y}_{\mathcal{S}}=y_{\mathcal{S}}(\hat{x}(\mathcal{S}))$ for every $\mathcal{S} \subseteq \mathcal{D}_{j} \cup \mathcal{A}_{j}$, where $\hat{x}\left(\mathcal{A}_{j}\right)=x\left(\mathcal{A}_{j}\right)$. If there is more than one such tuple, then it declares an error.

Analysis of the probability of decoding error. We first bound the probability $P_{1}$ that receiver $j$ declares an error in recovering $\left(y_{\{1\}}, \ldots, y_{[n]}\right)$, that is,

$$
\begin{gathered}
P_{1}=\mathrm{P}\left\{z\left(\hat{y}_{\{1\}}, \ldots, \hat{y}_{[n]}\right)=z\left(y_{\{1\}}, \ldots, y_{[n]}\right)\right. \\
\text { for some } \left.\left(\hat{y}_{\{1\}}, \ldots, \hat{y}_{[n]}\right) \neq\left(y_{\{1\}}, \ldots, y_{[n]}\right)\right\} .
\end{gathered}
$$

Since $\hat{y}_{\mathcal{S}}=y_{\mathcal{S}}$ for each $\mathcal{S} \subseteq \mathcal{A}_{j}$, the number of wrong tuples $\left(\hat{y}_{\{1\}}, \ldots, \hat{y}_{[n]}\right)$ is $2^{\sum_{\mathcal{S} Z \mathcal{A}_{j}} \gamma_{\mathcal{S}} t}-1$. Furthermore, the probability that two distinct indices are mapped to the same codeword is $1 / 2^{\beta t}$. Hence, by the union of the events bound,

$$
P_{1} \leq \frac{2^{\sum_{\mathcal{S} \notin \mathcal{A}_{j}} \gamma_{\mathcal{S}} t}}{2^{\beta t}},
$$

which tends to zero as $t \rightarrow \infty$, provided that $\beta>$ $\sum_{\mathcal{S} \nsubseteq \mathcal{A}_{j}} \gamma_{\mathcal{S}}$

Next, we bound the probability $P_{2}$ that receiver $j$ declares an error in recovering $\left(x_{1}, \ldots, x_{n}\right)$ from the correct tuple $\left(y_{\{1\}}, \ldots, y_{[n]}\right)$, that is,

$$
\begin{aligned}
P_{2}= & \mathrm{P}\left\{y_{\mathcal{S}}(\hat{x}(\mathcal{S}))=y_{\mathcal{S}} \text { for all } \mathcal{S} \subseteq \mathcal{D}_{j} \cup \mathcal{A}_{j}\right. \\
& \text { for some } \left.\left(\hat{x}_{1}, \ldots, \hat{x}_{n}\right) \neq\left(x_{1}, \ldots, x_{n}\right)\right\} .
\end{aligned}
$$

Since $\hat{x}\left(\mathcal{A}_{j}\right)=x\left(\mathcal{A}_{j}\right)$, we can decompose the wrong tuples $\hat{\mathbf{x}}:=\left(\hat{x}_{1}, \ldots, \hat{x}_{n}\right)$ by collecting the subset $\mathcal{T} \subseteq$ 
$\mathcal{D}_{j} \backslash \mathcal{A}_{j}$ of erroneous message indices, i.e., $\hat{x}_{i} \neq x_{i}$ iff $i \in \mathcal{T}$. Thus, by the union of the events bound, $P_{2}$ is upper bounded by

$$
\sum_{\mathcal{T} \subseteq \mathcal{D}_{j} \backslash \mathcal{A}_{j}} \sum_{\hat{\mathbf{x}}: \hat{x}_{i} \neq x_{i}, i \in \mathcal{T}} \mathrm{P}\left(\bigcap_{\substack{\mathcal{S} \subseteq \mathcal{D}_{\mathcal{j}} \cup \mathcal{A}_{j} \\ \mathcal{S} \cap \mathcal{T} \neq \emptyset}}\left\{y_{\mathcal{S}}(\hat{x}(\mathcal{S}))=y_{\mathcal{S}}\right\}\right) .
$$

Now for each $\mathcal{T}$ the number of wrong tuples is $\left(2^{t}-\right.$ $1)^{|\mathcal{T}|}$, and for each wrong tuple with $\hat{x}_{i} \neq x_{i}$ iff $i \in$ $\mathcal{T}$, the probability that two distinct message tuples are mapped to the same $y_{\mathcal{S}}$ for all $\mathcal{S} \subseteq \mathcal{D}_{j} \cup \mathcal{A}_{j}$ with $\mathcal{S} \cap \mathcal{T} \neq$ $\emptyset$ is $1 / 2^{\sum_{\mathcal{S} \subseteq \mathcal{D}_{j} \cup \mathcal{A}_{j}: \mathcal{S} \cap \mathcal{T} \neq \emptyset} \gamma_{\mathcal{S}} t}$. Hence,

$$
P_{2} \leq \sum_{\mathcal{T} \subseteq \mathcal{D}_{j} \backslash \mathcal{A}_{j}} \frac{2^{|\mathcal{T}| t}}{2^{\sum_{\mathcal{S} \subseteq \mathcal{D}_{j} \cup \mathcal{A}_{j}: \mathcal{S} \cap \mathcal{T} \neq \emptyset} \gamma_{\mathcal{S}} t}},
$$

which tends to zero as $t \rightarrow \infty$, provided that

$$
\frac{1}{|\mathcal{T}|} \sum_{\mathcal{S} \subseteq \mathcal{D}_{j} \cup \mathcal{A}_{j}: \mathcal{S} \cap \mathcal{T} \neq \emptyset} \gamma_{\mathcal{S}}>1, \quad \mathcal{T} \subseteq \mathcal{D}_{j} \backslash \mathcal{A}_{j} .
$$

\section{COMPARISON TO GRAPH COLORING CODING SCHEMES}

We show that the composite coding scheme (Theorem 3) outperforms the partial clique covering scheme (Theorem 1) and the fractional local graph coloring scheme (Theorem 2).

Theorem 4. $\beta_{\mathrm{CC}} \leq \beta_{\mathrm{PC}}$.

Proof: Given an index coding problem of side information graph $\mathcal{G}$, let $\mathcal{G}_{1}, \ldots, \mathcal{G}_{m}$ be partial cliques of parameters $k_{1}, \ldots, k_{m}$ that partition $\mathcal{G}$. For each $j$, let $\mathcal{D}_{j}=\mathcal{G}_{i}$ if $j \in \mathcal{G}_{i}$ and consider

$$
\gamma_{\mathcal{S}}= \begin{cases}k_{i}+1, & \text { if } \mathcal{S}=\mathcal{G}_{i} \text { for some } i, \\ 0, & \text { otherwise }\end{cases}
$$

By the definition of partial clique, if $\mathcal{D}_{j}$ is a $k\left(\mathcal{D}_{j}\right)$ partial clique,

$$
\left|\mathcal{D}_{j} \backslash \mathcal{A}_{j}\right| \leq k\left(\mathcal{D}_{j}\right)+1 .
$$

Moreover, for every $j \in[n]$ and every $\mathcal{T} \subseteq \mathcal{D}_{j} \backslash \mathcal{A}_{j}$,

$$
|\mathcal{T}| \leq\left|\mathcal{D}_{j} \backslash \mathcal{A}_{j}\right| \leq k\left(\mathcal{D}_{j}\right)+1=\sum_{\mathcal{S} \subseteq \mathcal{D}_{j} \cup \mathcal{A}_{j}: \mathcal{S} \cap \mathcal{T} \neq \emptyset} \gamma_{\mathcal{S}} .
$$

Hence, $\left(\gamma_{\mathcal{S}}: \mathcal{S} \subseteq[n]\right)$ is a feasible solution to (7) and

$$
\beta_{\mathrm{CC}} \leq \max _{j \in[n]} \sum_{\mathcal{S} \subseteq[n]: \mathcal{S} \nsubseteq \mathcal{A}_{j}} \gamma_{\mathcal{S}} \leq \sum_{\mathcal{S} \subseteq[n]} \gamma_{\mathcal{S}}=\beta_{\mathrm{PC}} .
$$

The inequality in Theorem 4 is sometimes strict. Consider the index coding problem in Example 1. For this problem, it can be checked that $\beta_{\mathrm{CC}}=5 / 2$ [15], which is equal to $\beta^{*}$, but $\beta_{\mathrm{PC}}=3$. (Note that $\beta_{\mathrm{PC}}$ is always an integer.)

Theorem 5. $\beta_{\mathrm{CC}} \leq \beta_{\mathrm{FL}}$.

Proof: Given an index coding problem, let $\rho_{\mathcal{S}}, \mathcal{S} \in$ $\mathcal{K}$, be the solution to the linear program in (4), i.e.,

$$
\beta_{\mathrm{FL}}=\max _{j \in[n]} \sum_{\mathcal{S} \in \mathcal{K}: \mathcal{S} \unrhd \mathcal{A}_{j}} \rho_{\mathcal{S}}
$$

and

$$
\sum_{\mathcal{S} \in \mathcal{K}: j \in \mathcal{S}} \rho_{\mathcal{S}} \geq 1, \quad j \in[n] .
$$

Let $\mathcal{D}_{j}=\{j\}, j \in[n]$, and consider

$$
\gamma_{\mathcal{S}}= \begin{cases}\rho_{\mathcal{S}}, & \text { if } \mathcal{S} \in \mathcal{K}, \\ 0, & \text { otherwise }\end{cases}
$$

Then,

$$
\begin{aligned}
\sum_{\mathcal{S} \subseteq[n]: \mathcal{S} \subseteq\{j\} \cup \mathcal{A}_{j}, j \in \mathcal{S}} \gamma_{\mathcal{S}} & =\sum_{\mathcal{S} \in \mathcal{K}: \mathcal{S} \subseteq\{j\} \cup \mathcal{A}_{j}, j \in \mathcal{S}} \rho_{\mathcal{S}} \\
& =\sum_{\mathcal{S} \in \mathcal{K}: j \in \mathcal{S}} \rho_{\mathcal{S}} \\
& \geq 1,
\end{aligned}
$$

where (9) follows since $j \in \mathcal{S} \in \mathcal{K}$ implies that $\mathcal{S} \subseteq$ $\{j\} \cup \mathcal{A}_{j}$. Hence, $\left(\gamma_{\mathcal{S}}: \mathcal{S} \subseteq[n]\right)$ is a feasible solution to (7) and

$\beta_{\mathrm{CC}} \leq \max _{j \in[n]} \sum_{\mathcal{S} \subseteq[n]: \mathcal{S} \unrhd \mathcal{A}_{j}} \gamma_{\mathcal{S}}=\max _{j \in[n]} \sum_{\mathcal{S} \in \mathcal{K}: \mathcal{S} \unrhd \mathcal{A}_{j}} \rho_{\mathcal{S}}=\beta_{\mathrm{FL}}$.

The inequality in Theorem 5 is sometimes strict, as demonstrated by the following.

Example 3. Consider the index coding problem

$$
(1 \mid 2,4,5,6,7),(2 \mid 3,4,5,6,7),(3 \mid 1,4,5,6,7),
$$

$(4 \mid 1,2,3,5,6,7),(5 \mid 2,3,6,7),(6 \mid 1,3,5,7),(7 \mid 1,2,5,6)$.

By solving the respective linear programs, it can be checked that $\beta_{\mathrm{CC}}=5 / 2<\beta_{\mathrm{FL}}=8 / 3$. Note that for this case, $\beta_{\mathrm{PC}}=3$.

\section{DISCUSSION}

The composite coding scheme in this paper can be generalized in several directions. First, it can be extended to the broadcasting with side information problem studied by Alon, Hassidim, Lubetzky, Stav, and Weinstein [5], in which each message can be requested by more than one receiver. In addition, it can be extended to the variable message rate case studied by Blasiak, Kleinberg, and Lubetzky [27], in which each message $x_{j}$ has its individual rate $\beta_{j}$. The optimal tradeoff between these individual rates are better captured by that between their reciprocals (which correspond to the 
capacity region in network coding problems). In the expanded version of the paper to be posted in arXiv, we will discuss the information-theoretic formulation of this problem (including multiple receivers), the general composite coding scheme and the resulting inner bound on the capacity region, and an outer bound on the capacity region based on submodularity of entropy.

Example 3 demonstrated that the composite coding scheme can strictly outperform the better of the partial clique covering and fractional local graph coloring schemes. Let $\mathcal{G}$ be the union of the corresponding side information graph $\mathcal{G}$ and consider the $n$-blow-up of $\mathcal{G}$, namely, the Cartesian product of $\mathcal{G}$ and the graph $([n], \emptyset)$. The additivity of the optimal broadcast rate for Cartesian product implies that there is an $\Theta(n)$ gap between $\beta_{\mathrm{CC}}$ and $\min \left\{\beta_{\mathrm{PC}}, \beta_{\mathrm{FL}}\right\}$. It remains open to prove or disprove that this gap can be strengthened to a multiplicative one.

The following example, in turn, demonstrates that the composite coding scheme is suboptimal.

Example 4. Consider the 7-message index coding problem

$$
\begin{gathered}
(1 \mid 2,3,4,6,7),(2 \mid 1,3,6,7),(3 \mid 1,4,5,7),(4 \mid 1,2,5,6), \\
(5 \mid 3,4,6,7),(6 \mid 2,4,5,7),(7 \mid 2,3,5,6) .
\end{gathered}
$$

Using the vector linear coding scheme built on interference alignment, Jafar [11] showed that $\beta^{*}=5 / 2$. In comparison, it can be shown that $\beta_{\mathrm{CC}}=8 / 3$.

Thus, there is a $\Theta(n)$ additive gap between $\beta^{*}$ and $\beta_{\mathrm{CC}}$ in general. Again there is no known multiplicative gap between $\beta^{*}$ and $\beta_{\mathrm{CC}}$.

\section{REFERENCES}

[1] Y. Birk and T. Kol, "Informed-source coding-on-demand (ISCOD) over broadcast channels," in Proc. 17th Ann. IEEE Int. Conf. Comput. Commun. (INFOCOM), San Francisco, CA, Mar. 1998, pp. 1257-1264.

[2] _ - "Coding on demand by an informed source (ISCOD) for efficient broadcast of different supplemental data to caching clients," IEEE Trans. Inf. Theory, vol. 52, no. 6, pp. 2825-2830, Jun. 2006.

[3] Z. Bar-Yossef, Y. Birk, T. S. Jayram, and T. Kol, "Index coding with side information," IEEE Trans. Inf. Theory, vol. 57, no. 3, pp. 1479-1494, Mar. 2011.

[4] E. Lubetzky and U. Stav, "Nonlinear index coding outperforming the linear optimum," IEEE Trans. Inf. Theory, vol. 55, no. 8, pp. 3544-3551, Aug. 2009.

[5] N. Alon, A. Hassidim, E. Lubetzky, U. Stav, and A. Weinstein, "Broadcasting with side information," in 49th Ann. IEEE Symp. Found. Comput. Sci., Philadelphia, PA, Oct. 2008, pp. 823-832.

[6] A. Blasiak, R. Kleinberg, and E. Lubetzky, "Broadcasting with side information: Bounding and approximating the broadcast rate," IEEE Trans. Inf. Theory, vol. 59, no. 9, pp. 5811-5823, Sep. 2013.

[7] S. El Rouayheb, A. Sprintson, and C. Georghiades, "On the index coding problem and its relation to network coding and matroid theory," IEEE Trans. Inf. Theory, vol. 56, no. 7, pp. 3187-3195, Jul. 2010.
[8] M. Effros, S. El Rouayheb, and M. Langberg, "An equivalence between network coding and index coding," 2012. [Online]. Available: http://arxiv.org/abs/1211.6660/

[9] H. Maleki, V. R. Cadambe, and S. A. Jafar, "Index coding: An interference alignment perspective," in Proc. IEEE Int. Symp. Inf. Theory, Cambridge, MA, Jul. 2012, pp. 2236-2240.

[10] K. Shanmugam, A. G. Dimakis, and M. Langberg, "Local graph coloring and index coding," in Proc. IEEE Int. Symp. Inf. Theory, Istanbul, Turkey, Jul. 2013, pp. 1152-1156.

[11] S. A. Jafar, "Topological interference management through index coding," 2013. [Online]. Available: http://arxiv.org/abs/1301.3106/

[12] N. Naderializadeh and A. S. Avestimehr, "Interference networks with no csit: Impact of topology," 2013. [Online]. Available: http://arXiv.org/abs/1302.0296/

[13] M. Langberg and M. Effros, "Network coding: Is zero error always possible?" in Proc. 49th Ann. Allerton Conf. Comm. Control Comput., Monticello, IL, Sep. 2011, pp. 1478-1485.

[14] M. A. Maddah-Ali and U. Niesen, "Fundamental limits of caching," 2012. [Online]. Available: http://arxiv.org/abs/1209.5807/

[15] F. Arbabjolfaei, B. Bandemer, Y.-H. Kim, E. Sasoglu, and L. Wang, "On the capacity region for index coding," in Proc. IEEE Int. Symp. Inf. Theory, Istanbul, Turkey, Jul. 2013, pp. 962 966.

[16] R. Ahlswede, N. Cai, S.-Y. R. Li, and R. W. Yeung, "Network information flow," IEEE Trans. Inf. Theory, vol. 46, no. 4, pp. 1204-1216, 2000.

[17] C. E. Shannon, "A mathematical theory of communication," Bell Syst. Tech. J., vol. 27, no. 3, pp. 379-423, 27(4), 623-656, 1948.

[18] T. M. Cover, "A proof of the data compression theorem of Slepian and Wolf for ergodic sources," IEEE Trans. Inf. Theory, vol. 21, no. 2, pp. 226-228, Mar. 1975.

[19] F. Arbabjolfaei, B. Bandemer, and Y.-H. Kim, "Optimal broadcast rates and capacity regions for index coding problems with up to 5 messages." [Online]. Available: http://circuit.ucsd.edu/ yhk/indexcoding.html

[20] F. J. MacWilliams and N. J. A. Sloane, The Theory of ErrorCorrecting Codes. Amsterdam: North-Holland, 1977.

[21] P. Erdös, Z. Füredi, A. Hajnal, P. Komjáth, V. Rödl, and Á. Seress, "Coloring graphs with locally few colors," Discrete Math., vol. 59, no. 1, pp. 21-34, 1986.

[22] J. Körner, C. Pilotto, and G. Simonyi, "Local chromatic number and Sperner capacity," J. Combin. Theory Ser. B, vol. 95, no. 1, pp. 101-117, 2005.

[23] M. A. Maddah-Ali, A. S. Motahari, and A. K. Khandani, "Communication over MIMO X channels: Interference alignment, decomposition, and performance analysis," IEEE Trans. Inf. Theory, vol. 54, no. 8, pp. 3457-3470, 2008.

[24] V. R. Cadambe and S. A. Jafar, "Interference alignment and degrees of freedom of the $K$-user interference channel," IEEE Trans. Inf. Theory, vol. 54, no. 8, pp. 3425-3441, Aug. 2008.

[25] T. M. Cover and J. A. Thomas, Elements of Information Theory, 2nd ed. New York: Wiley, 2006.

[26] A. El Gamal and Y.-H. Kim, Network Information Theory. Cambridge: Cambridge University Press, 2011.

[27] A. Blasiak, R. Kleinberg, and E. Lubetzky, "Index coding via linear programming," 2011. [Online]. Available: http://arxiv.org/abs/1004.1379 